\title{
Le cycle biologique de Cyclops vicinus vicinus (Copepoda, Cyclopoida) dans la retenue de Serre-Ponçon (Hautes-Alpes)
}

\author{
Y. Bressacl \\ A. Champeau 1
}

L'étude du cycle biologique de Cyclops vicinus vicinus à trois stations du réservoir de Serre-Ponçon révèle l'existence d'une hétérogénéité du milieu, tant au niveau des effectifs que du nombre de générations annuelles. Les variations observées s'expliquent par des phases de vie latente (diapause ou quiescence) dont le déterminisme est discuté.

The life cycle of Cyclops vicinus vicinus (Copepoda, Cyciopoida) in the reservoir of Serre-Ponçon (High Alps).

A study of the life cycle of Cyclops vicinus vicinus at three stations in the reservoir of Serre-Ponçon showed that the heterogeneity of the environment was sufficient to effect the number of generations per year. The observed variations could be explained by the phases of resting stages (diapause or quiescence) and the reasons for this are discussed.

\section{Introduction}

Dans le cadre d'une étude hydrobiologique du lac de barrage de Serre-Ponçon, l'examen du zooplancton a permis de mettre en évidence la présence de trois espèces dominantes: Daphnia longispina, Acanthodiaptomus denticomis, Cyclops vicinus vicinus (Bressac 1982).

L'objet de ce travail est de décrire le cycle biologique de $C$. vicinus en suivant l'èvolution des effectifs des différents stades ontogéniques en activité dans la masse d'eau et en vie latente dans le sédiment et de tenter de mettre en relation l'évolution saisonnière de ces effectifs avec les conditions du milieu (paramètres physiques et chimiques, bacté. riologie, phytoplancton).

\section{1. - Le milieu}

Serre-Ponçon, avec une superficie de 2894 ha, est le plus grand lac de barrage français. Il est situé à une altitude de $780 \mathrm{~m}$ et sa profondeur maximale atteint $120 \mathrm{~m}$. Il présente une double alimentation par les rivières Durance et Ubaye, un temps de

1. Laboratoire d'Hydrobiologie, Université de Provence, 1 place Victor Hugo, 13331 Marseille Cedex 3. renouvellement de l'eau relativement court (150 jours) et un marnage saisonnier important (baisse de niveau de 30 à $50 \mathrm{~m}$ en hiver). C'est un milieu jeune (mise en eau en 1960)

Deux stations aux caractéristiques différentes ont été choisies pour rendre compte de l'hétérogénéité du peuplement zooplanctonique de cette retenue, hétérogénéité fréquente dans les lacs de grande taille (Patalas 1981). La station 1, "Savines", est situee en queue de retenue, à faible distance du débouché de la Durance. La profondeur est de $40 \mathrm{~m}$ Iorsque le niveau du lac est au plus haut. La station 2, "Barrage», est située dans la zone la plus profonde $(120 \mathrm{~m})$ et la plus éloignée de l'arrivée des rivières (fig. 1).

\section{2. - Méthodes de prélèvement}

L'échantillonneur employé pour les 26 campagnes. échelonnées de 1978 à 1981 est un appareil de type Clarke-Bumpus. Il est muni d'un filet de 125 $\mu$ de vide de maille et tracté horizontalement à une vitesse de $120 \mathrm{~m} / \mathrm{mn}$ sur un parcours de $300 \mathrm{~m}$ décrivant un "aller-retour" (Bressac 1982).

Les traits sont effectués aux profondeurs $0-5,5 \cdot 10$, $10-20,20-40,40-60$, et $60-100 \mathrm{~m}$ à "Barrage " et dans les trois premières de ces couches d'eau à «Savines". Les résultats exprimés en nombre d'individus par 


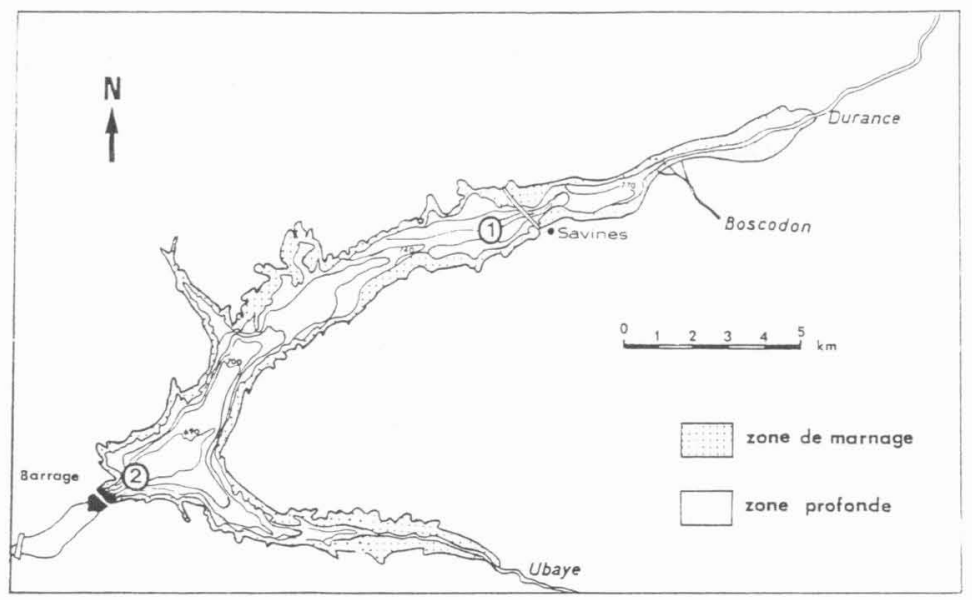

FIG. I. La retenue de Serre-Ponçon. 1: Station "Savines"; 2 : Station "Barrage»

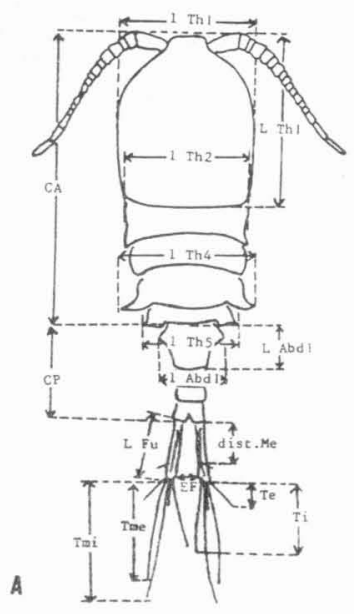

Fig. 2. Détermination du cyclopide de Serre-Ponçon: A - Mensurations utilisées pour la détermination des espèces du genre Cyclops.

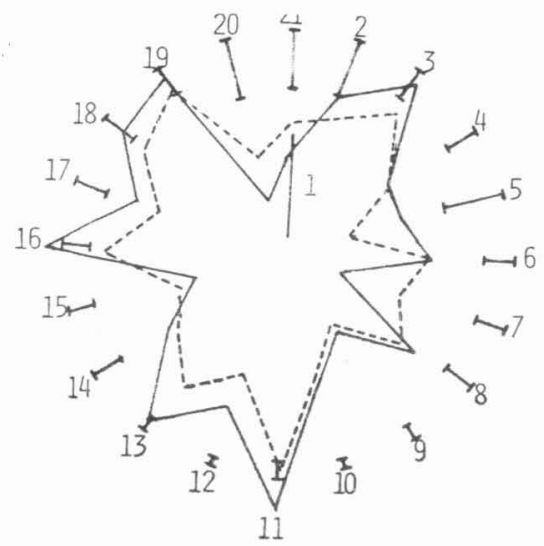

B B-Diagrammes polaires: - obtenu pour les individus récoltés à Serre-Ponçon ;...donné par Dussart (1969) pour l'espèce C. vicinus vicinus.

L'échelle est différente sur chaque axe et représentée sur le cercle exieme par un segment correspondant à un rapport de 0,1 . 
$\mathrm{m}^{3}$, correspondent à une moyenne pondérée sur l'ensemble de la colonne d'eau.

Les résultats concernant le nombre de copépodites en vie latente au niveau du fond, à l'aplomb des stations prospectées pour le zooplancton, sont exprimés en nombre d'individus par $\mathrm{m}^{2}$ de sédiment. L'échantillonnage a été effectué avec une benne d'Eckmann et testé par Nino (1982).

\section{3. - Détermination du cyclopide}

La détermination jusqu'à l'espèce des individus du genre $C y c l o p s$ nécessite une vingtaine de mensurations sur un lot d'au moins 10 femelles. Les variations individuelles de taille obligent à considérer non pas directement ces mensurations mais leurs rapports deux à deux. Ces rapports sont comparés les uns aux autres en les groupant sur un diagramme en coordonnées polaires qui permet d'intégrer l'ensemble des caractères observés. Ce diagramme est établi d'après une grille donnée par Dussart (1958) et comparé à ceux obtenus par cet auteur pour les différentes espèces du genre Cyclops (Fig. 2).

Le diagramme obtenu pour les individus récoltés à Serre-Ponçon se rapproche de celui réalisé par Dussart pour $C$. vicinus vicinus. De plus, les rapports des mesures sont comparables à ceux donnés par Origgi et col. (1978) et les individus de Serre.Ponçon correspondent à la description de Einsle (1975).

Cependant des variations morphologiques d'origine phénotypique et/ou génotypique peuvent affecter les différentes populations d'une mème espèce de Cyclops, si bien que le diagramme obtenu à SerrePonçon n'esı pas superposable à celui donné par Dussart. Dans ces conditions, il est utile de faire état des mensurations pour caractériser les popula ons de Serre-Ponçon (Tableau I).

Tableau I. Valeurs des rapports calculés a partir des mensurations des cyclopides de Serre.Ponçon.

\begin{tabular}{|c|c|c|}
\hline Axe & Parametres mesurés & Rapport \\
\hline 1 & LT & $1.76 \mathrm{~mm}$ \\
\hline 2 & CPICA & 0.43 \\
\hline 3 & L. Thll Thl & 1,22 \\
\hline 4 & | Th2/ Th| & 0,91 \\
\hline 5 & L AbdI// Abd1 & 0,95 \\
\hline 6 & L Fu/l Fu & 5,86 \\
\hline 7 & EF/L Fu & 0,09 \\
\hline 8 & $\mathrm{~T} \mathrm{me} / \mathrm{T} \mathrm{mi}$ & 0,85 \\
\hline 9 & TetL Fu & 0,54 \\
\hline 10 & $\mathrm{~T}$ me/L Fu & 1.87 \\
\hline 11 & $\mathrm{Ti} / \mathrm{Te}$ & 2,40 \\
\hline 12 & $\mathrm{~T} \mathrm{mi} / \mathbf{L} \mathbf{F u}$ & 2.18 \\
\hline 13 & Ti/L Fu & 1,32 \\
\hline 14 & $\operatorname{dis} 1 M . L F u$ & 0.75 \\
\hline 15 & ExtP4:IntPa & 2,29 \\
\hline 16 & I Th5! Abd1 & 1.49 \\
\hline 17 & I Th5/] Th4 & 0,82 \\
\hline 18 & I Th5/1 Th1 & 0.67 \\
\hline 19 & I Th4lf Th1 & 0,81 \\
\hline 20 & I CptuLT & 0.22 \\
\hline 21 & L Cph/LT & 0,207 \\
\hline
\end{tabular}

\section{4. - Cycle biologique de Cyclops vicinus vicinus aux deux stations étudiées}

\section{1 - Résultats}

L'analyse détaillée des échantillons porte sur les prélèvements de 1980 et du début de 1981 .

Pour le cycle annuel de 1980, le profil géneral de l'évolution saisonnière des différents stades ontogéniques des cyclopides est équivalent aux deux sta-

Tableau II. Densités des différents stades ontogéniques de C. cicimus vicintus à la station 1, "Savines", en 1980 et 1981 (numbre d'individus par $\mathrm{m}^{3}$ ).

\begin{tabular}{|c|c|c|c|c|c|c|c|c|c|c|c|c|c|c|c|}
\hline stade. & $10 / 0 t$ & 08105 & $10 \% 06$ & $17 / 06$ & $10 / 07$ & 27107 & $08 / 08$ & $22 / 08$ & $04 / 09$ & $17 / 09$ & $08^{\prime} 10$ & $04 / 12$ & $25 / 03$ & $06 / 05$ & $10 / 06$ \\
\hline natu. & 540 & 2468 & 150 & 20 & 121 & 40 & 152 & 540 & 73.5 & 2355 & 718 & 2768 & 7077 & 4452 & 275 \\
\hline C. 1 & 440 & 3753 & 112 & 20 & 64 & 122 & 50 & 450 & 353 & 1920 & 663 & 2365 & 4684 & 1985 & 282 \\
\hline C. 2 & 380 & 4360 & 50 & 24 & 43 & 27 & 5 & 265 & 275 & 740 & 311 & 1345 & 3272 & 2599 & 182 \\
\hline C. 3 & 360 & 2050 & 20 & 5 & 65 & 23 & 7 & 85 & 145 & 422 & 358 & 675 & 2903 & 6419 & 175 \\
\hline C. 4 & 520 & 561 & 177 & 8 & 38 & 50 & 32 & 95 & 118 & 320 & 462 & 809 & 2659 & 1697 & 152 \\
\hline C. 5 & 200 & 566 & 282 & 24 & 10 & 7 & 11 & 38 & 103 & 70 & 407 & 461 & 1045 & 1040 & 80 \\
\hline$\sigma$ & 180 & 257 & 35 & 25 & 11 & 10 & 147 & 210 & 63 & 180 & 567 & 716 & 968 & 802 & 157 \\
\hline$\rho$ & 120 & 535 & 157 & 43 & 56 & 36 & 95 & 130 & 60 & 76 & 730 & 248 & 488 & 484 & 192 \\
\hline Qw & . & 42 & 20 & 6 & 40 & 13 & 61 & 3.3 & 41 & 15 & 122 & 57 & 70 & 17 & 59 \\
\hline W & - & 907 & 345 & 70 & 837 & 245 & 2291 & 1210 & 1147 & 190 & 3491 & 1460 & 1936 & 375 & 1000 \\
\hline Total & 2740 & 14783 & 985 & 173 & 418 & 31.5 & 500 & 1820 & 1860 & 6086 & 4420 & 9381 & 23070 & 20048 & 1535 \\
\hline
\end{tabular}


tions (Tableau II et fig. 3). La densité de la population est très faible en hiver. Tous les stades de déve. loppement présentent un pic d'abondance au printemps (prélèvements de mai), très fortement mar. qué pour les individus jeunes (nauplies et copépodites 1 à 3). Une chute brutale des effectifs de tous les stades est enregistrée début juin, après la crue.

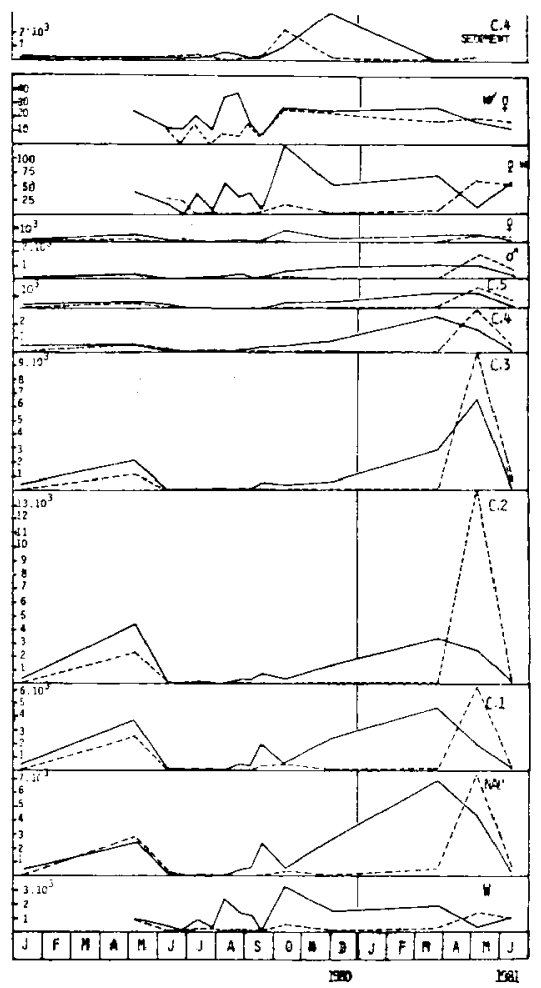

Fig. 3. Evolution saisonnière des densités des différents stades ontogéniques de $C$. vicinus vicinus aux stations "Savines" et a Barrage* en 1980 et 1981 :

- dans le sédiment (C.4 = copépodites 4 en vie latente); - dans la masse d'eau $(w=$ cufs ; nau = nauplies $: C .1$ à $\mathrm{C} .5=$ copépodites 1 à $5 ; \sigma=$ adultes mâles ; $9=$ adultes femelles; $\uparrow w=$ femelles ovigères.
Les cyclopides demeurent très peu représentés dans la masse d'eau tout au long de l'été. Ce n'est qu'en septembre que les densités des différents stades augmentent de nouveau à “Savines» comme à "Barrage".

Cependant, des différences importantes se manifestent entre les deux stations. Tout d'abord, pour la quasi totalité des campagnes, la quantité de cyclopides récoltés est nettement supérieure à la station 1, "Savines", qu'à la station 2, "Barrage" (en moyenne 2,5 fois plus). D'autre part, au cours de l'été et bien que les effectifs de tous les stades restent faibles aux deux stations, une augmentation de ces effectifs est notée à la station 1 début juillet pour les œufs et les stades jeunes (nauplies et copépodites 1 à 4), début août pour les adultes, ceufs, stades naupliens et copépodites 1 , fin août et début septembre pour l'ensemble des stades. En revanche, à la station 2, "Barrage", les densités demeurent très faibles jusqu'à la fin septembre.

Les périodes de reproduction, marquées par les pics d'abondance de femelles ovigères et par l'augmentation du nombre d'cufs par femelle confir. ment cette différence. Elles se manifestent au début des mois de mai, juillet, août et septembre et à la fin d'octobre à la station 1. A la station 2, en revanche, si deux phases sont aussi bien marquées en mai et en octobre, une seule est en registrée au cours de l'été, début août (Tableaux II et III, fig. 3).

Enfin, les arrêts de croissance dans le cycle biologique de cette espèce se signalent par l'augmentation du nombre de copépodites $(0,5 \%$ de copépodites $3 ; 98,5 \%$ de copépodites $4 ; 1 \%$ de copépodites 5) récoltés dans les sédiments avec la faune benthique. Deux phases nettes sont marquées pour chacune des deux stations:

- à la station "Savines» en août $\left(13.10^{3}\right.$ copépodites $\left./ \mathrm{m}^{2}\right)$ et décembre $\left(105.10^{3}\right.$ copépodites $\left./ \mathrm{m}^{2}\right)$;

- à la station "Barrage» en juillet $\left(24.10^{3}\right.$ copépodites $\left./ \mathrm{m}^{2}\right)$ et en octobre $\left(45.10^{3}\right.$ copopédites $\left./ \mathrm{m}^{2}\right)$.

Les trois campagnes du début 1981 soulignent la variabilité spatio-temporelle de l'évolution saisonnière de la population de cyclopides à Serre-Ponçon. Dans le temps, à la station «Savines» où le prélèvement d'hiver comporte, en 1981, un effectif très important $(3,4$ fois plus d'individus que pour le prélèvement de l'hiver précédent). Les femelles ovigères sont très nombreuses à cette date, mais les individus les mieux représentés appartiennent aux sta- 
Tableau 1II. Densités des différents stades ontogéniques de C. vicinus vicinus à la station 2, "Barrage*, en 1980 et t981 (nombre d'individus par $\mathrm{m}^{3}$ ).

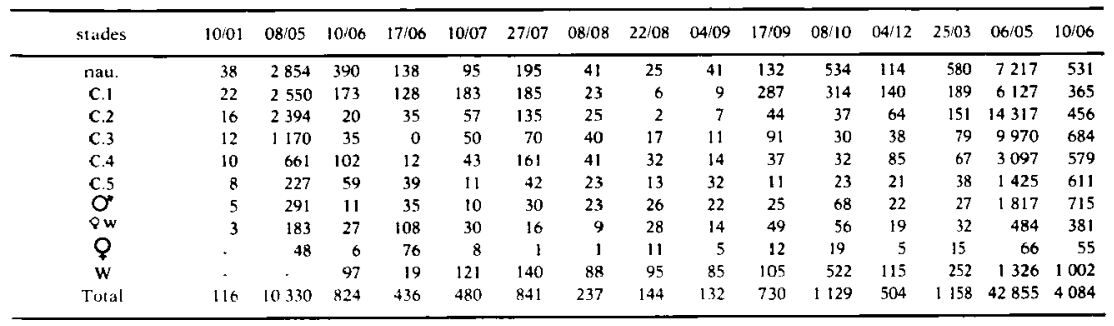

des les plus jeunes (nauplies et copépodites 1 et 2). Au printemps, la prolifération de tous les stades, notée en mai 1980, est remplacée en 1981 par une "explosion" de l'espece aux deux stations avec jusqu'à $42,8.10^{3}$ ind. $/ \mathrm{m}^{3}$ (à «Barrage», en mai) pour seulement $10,3,10^{3}$ ind $/ \mathrm{m}^{3}$ l'année précédente.

Dans le temps, enfin, cette prolifération de l'espèce au début 1981 est enregistrée plus tôt à a Savines" (mars) qu'à "Barragen (mai).

\section{2 Interprétation}

D'après ces résultats une interprétation du cycle biologique de $C$. vicinus vicinus à chacune des stations peut être faite sur le modèle de celles proposées par Einsle (1975) pour C. abyssorum et par Elg. mork \& Langeland (1980) pour $G$. scutifer. Les différences notées dans l'évolution saisonnière des différents stades ontogéniques peuvent étre la traduction du développement d'un nombre différent de générations annuelles à l'une et à l'autre des stations.

- A la station 1, "Savines" (fig. 4)

Cinq générations semblent se succéder.

la génération 1 est issue des pontes (z) des femelles de la dernière génération de l'année précédente (Z). Ces pontes se produisent à la fin de l'hiver, jusqu'en mai. Au cours du mois de juin, les individus de cette génération qui n'ont at teint pour la plupart que le stade copépodite 3 disparaissent au cours de la crue printanière. Seules quelques femelles sont encore récoltées à la fin de ce mois. Elles produisent des œufs début juillet (ponte a). $60 \%$ des femelles " 1 " sont ovigères mais le nombre d'œufs par femelle reste faible (15 à 20 ).
La génération 2 a un effectif très réduit. La sexratio ne se rétablit que fin juillet. Une nouvelle phase de ponte est enregistrée début août (b). Le nombre moyen d'ceufs par femelle passe de 15 (fin juillet) à 35 .

La génération 3, mieux représentée, se reproduit début septembre. La quantité de femelles ovigères a un peu diminué ainsi que le nombre d'œufs par femelle (ponte c). Cependant la population de $C$. vicinus vicinus est très abondante en septembre.

La génération 4 produit un très grand nombre d'œufs (ponte c) en octobre.

La génération 5 constitue la population hivernale. Une grande partie de ces individus entrent en diapause en décembre.

\section{- A la station "Barrage" (fig. 5)}

Il semble que trois générations seulement puissent être décomptées en 1980. La majorité des individus de $Z$, génération de 1979, a passé l'hiver en vie latente dans les sédiments au stade copépodite 4 (16. $10^{3}$ copépodites $/ \mathrm{m}^{2}$ en mai). Ils émergent et terminent leur développement au début du printemps 1980 et se reproduisent en mars-avril (ponte z).

La génération 1 développe des effectifs importants en mai, mais la population est décimée début juin pendant la période de crue. De plus, la majorité des copépodites 4 entrent en diapause (forte augmentation des effectifs dans les sédiments). Ils émergent progressivement au cours de juillet et jusqu'en août. Les premiers qui atteignent le stade adulte commencent à se reproduire fin juillet. Cette phase de ponte se poursuit jusqu'à la fin août.

La génération 2 issue de cette ponte étalée dans le temps n'est représentée au sein de la masse d'eau 


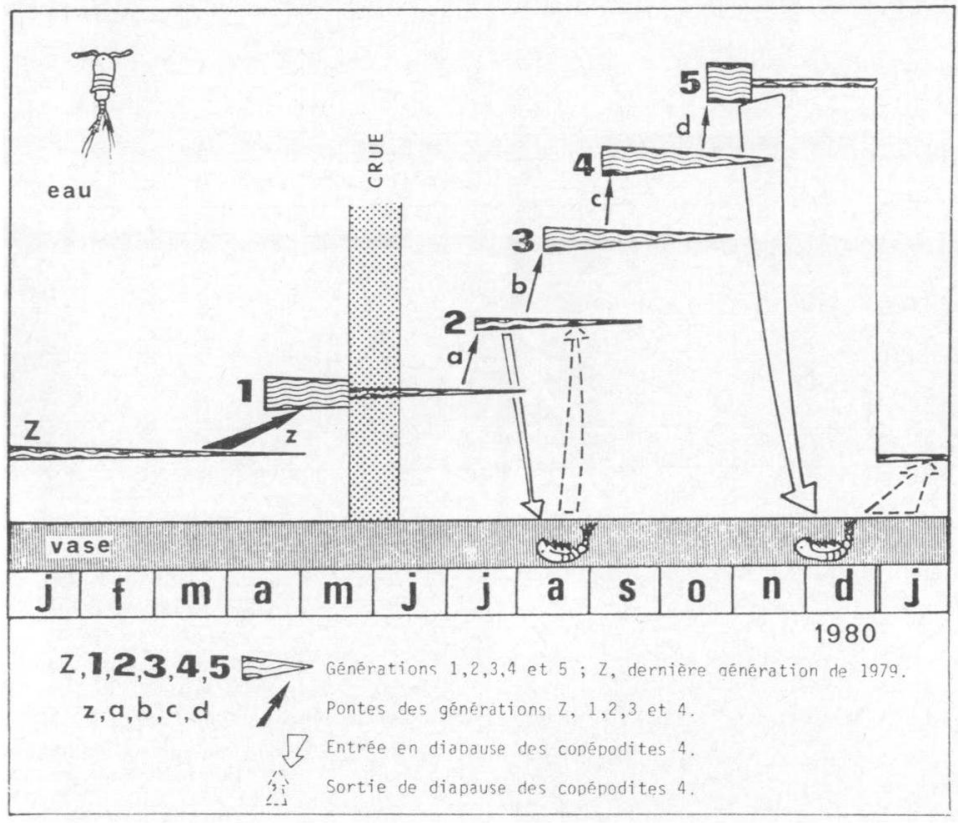

FIG. Interprétation du cvcle biologique de Cyclops vicinus vicintss a la station "Savines" en 1980 .

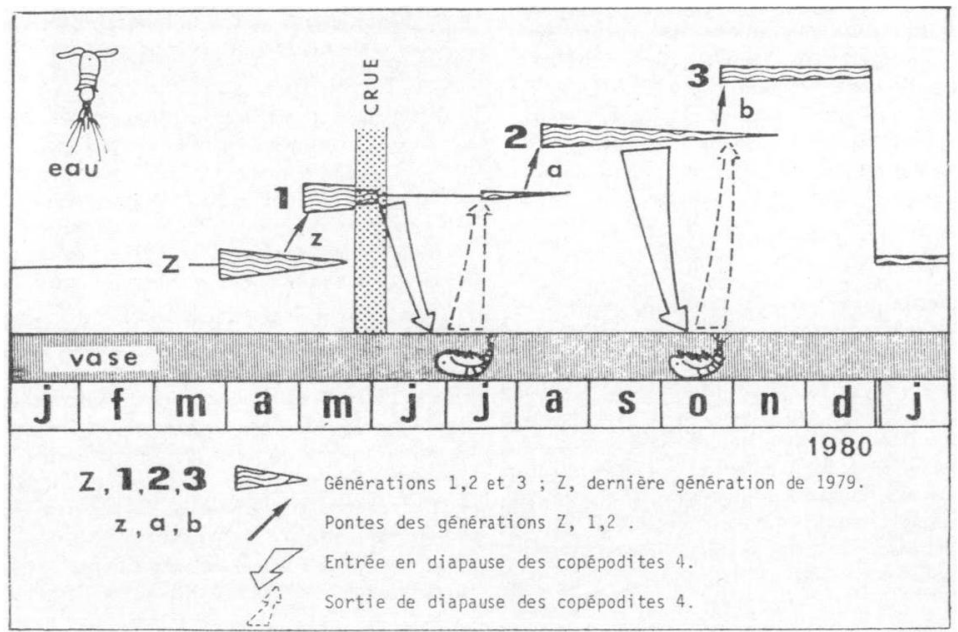

FiG. 5. Interprétation du cycle biologique de Cyclops vicinus vicinus à la station *Barrage» en 1980. 
que par des effectifs réduits. En effet les copépodi. tes qui arrivent successivement au stade 4 entrent en diapause et sont stockés au niveau du fond. C'est pourquoi la densité des copépodites au fond augmente tout au long de l'été pour atteindre son maxjmum début octobre. A cette époque une forte proportion de copépodites sortent en même temps de diapause et finissent leur développement pour se reproduire au cours du mois d'octobre.

La génération 3 se développe au cours de l'hiver.

\section{5. - Discussion}

Cyclops vicinus vicinus Uljanin, 1875 est une espèce cosmopolite, très commune dans le plancton d'Europe et d'Asie (Gurney 1933, Lindberg 1957, Rylov 1963, Dussart 1969). Elle est typique du plancton pélagique et réputee pour sa grande tolérance aux modifications de la composition de l'eau (Origgi \& al. 1978).

Les données de la littérature montrent que cette espèce présente, selon les milieux où elle est récoltée:

- une seule génération, au printemps (Hutchinson 1967):

- deux générations, une au printemps, l'autre à l'automne (Dussart 1969);

quatre ou cinq générations selon les années (George 1976).

Il ressort des résultats exposés çi-dessus que, pour un même écosystème, la retenue de Serre-Ponçon, la population de $C$. vicintus vicinus peut présenter d'importantes variations d'effectifs et du nombre de générations annuelles d'une station à l'autre et d'une année à l'autre puisque, au cours du cycle annuel de 1980, cinq générations semblent se succéder à "Savines", avec un nombre de cyclopides en moyenne 2,5 fois supérieur à celui récolté à "Barrage " où seulement trois générations peuvent être décomptées.

Ces résultats se retrouvent au niveau des autres composantes du zooplancton (le copépode calanoide Acanthodiaptomus denticornis, le cladocère Daph. nia longispina et le rotifère Asplanchna priodonta), toujours mieux représentés à "Savines" (Bressac 1983). Il en est de même pour les peuplements phytoplanctonique et bactérien qui présentent des densités supérieures à "Savines" en 1980 et supérieures aux deux stations au début 1981 par rapport aux prélèvements des années précédentes.
La période de crue de juin 1980 est nettement marquée avec des teneurs importantes en matières en suspension : de 2 à $12 \mathrm{mg} / \mathrm{l}$, selon la profondeur, à "Savines"; de 0.6 à $2.5 \mathrm{mg} / \mathrm{l}$ à "Barrage». De plus, le taux de saturation en oxygène dissous passe, à cette date, de $110 \%$ en surface à moins de $80 \%$ à $2,5 \mathrm{~m}$ et chute jusqu'à des valeurs de 40 à $50 \%$ sur le reste de la colonne d'eau, à «Savines". Cette désaturation est moins importante à "Barrage" puisqu'elle ne descend pas au dessous de $67 \%$ (Bagarry 1982). Ces fortes teneurs en matières en suspension, réputées préjudiciables au développement des organismes filtreurs que sont les jeunes cyclopides (Zurek 1980), associés à la chute brutale du taux d'oxygène peuvent contribuer à la forte mortalité de $C$. vicinus, en juin 1980.

Ce phénomène ne se reproduit pas l'année suivante. En effet, la période et la durée de remplissage du lac, au printemps, sont très variables d'une année a l'autre. En juin 1980, le niveau remonte de $15 \mathrm{~m}$ en 20 jours alors que la crue se manifeste dès mars en 1981 où elle est plus étalee dans le temps : le niveau remonte de $15 \mathrm{~m}$ en deux mois.

Pour le reste du cycle annuel, les valeurs mesu. rées pour les paramètres physiques et chimiques sont très peu différentes d'une station à l'autre. Le lac est caractérisé par des teneurs faibles en sels nutritifs : moins de $0.01 \mathrm{mg} / \mathrm{d}$ dorthophosphates pour tous les prélèvements; de $0,2 \mathrm{mg} / 1$ pour les nitrates à l'automne à $1,3 \mathrm{mg} / \mathrm{l}$ au printemps, aux deux stations (Bagarry 1982).

Cependant les densités bactériennes, phyto et zooplanctoniques plus élevées en queue de retenue laissent supposer que la quantité de sels nutritifs apportés par la Durance est plus élevée à "Savines" qu'au barrage et plus élevée en 1981 que les autres années. Mais cette différence n'apparait pas dans les dosages, les éléments nutritifs pouvant être intégrés à la matière vivante au fur et à mesure de leur disponibilité (Rigler 1973).

Au niveau du cycle biologique de Cyclops vicinus vicinus l'hétérogénéité du milieu peut se traduire aussi par le développement d'un nombre variable de générations annuelles. Les états de vie latente (en diapause ou en quiescence) à un stade copépoditique (copépodites 4 en majorité) permettent de réguler le nombre de générations du cyclopide.

Ainsi les résultats obtenus à Serre-Ponçon montrent que $C$. vicinus vicinus est une espèce capable de répondre à la variabilité des conditions de ce 
milieu artificiel. La diapause apparaît alors pour cette espèce comme un moyen permettant de moduler le cycle biologique en relation avec les ressources alimentaires disponibles.

\section{Travaux cités}

Bagarry (C.). 1982.- Contribution a l'élude d'un lac de barrage, la retenue de Serre-Ponçon : le bassin versant, l'évolution des paramètres abiotiques. Thése 3 cycle, Lniversité Aix-Marseille I, $141 \mathrm{p}$.

Bressac (Y.). 1982. - Contribution à l'étude du peuplement zooplanctonique d'un lac de barrage : la retenue hydroélectrique de Serre-Ponçon. Thèse de 3* cycle, Université Aix-Marseille I, $153 \mathrm{p}$.

Bressac (Y.). 1983. - Evolution saisonnière et pluriannuelle du peuplement zooplanctonique de la retenue de Serre-Ponçon. Biol. Ecol. Medit. A paraitre.

George (D.G.). 1976.- Life cycle and production of Cyclops vicinus in a shallow eutrophic reservoir. Oikos, $27: 101-110$.

Gurney (R.). 1983. - British freshwater copepoda. Roy Soc. London III : $234-248$.

Hutchinson (G.E.). 1967. - A treatise on Limnology. Vol. II. Introduction to lake biology and the limnoplankton. John Wiley sons, inc. New York.

Dussart (B.). 1958 - Remarques sur le genre Cyclops s. str. (Crust. Cop.). Hydrobiologia, $10: 263-292$.

Dussart (B.). 1969. - Les Copépodes des eaux continentales d'Europe occidentale. T. II : Cyclopoides et Biologie. Ed. N. Boubee et Cie : 292 p
Einsle (U.) 1967- Die äusseren Bedingungen der Diapause planktisch-lebender Cyclops-Arten. Arch. Hydrobiol. 63 (3): 387.403 .

Einsle (U.). 1975. - Revision der Gattung Cyclops s. str. speziell der aby'ssorum Gruppe. Mem. Ist. Ilat. Idrobiol. 32 : $57-219$

Elgmork (K.) \& Langeland (A.). 1980.-Cyclops scutifer Sars-One and two year life cycles with diapause in the meromictic lake Blankvatn. Arch. Hydrobiol. 88,2: $178 \cdot 201$

Lindberg (K.) 1957 - Le groupe Cyclops rubens (syn. C strenutus, genre Cyclops s. str. (O.F. Muller, 1770) C.W.K. Gleerup. Lund. 335 p.

Nino (A.). 1982 - La faune benthique du lac de barrage de Serre Ponçon : structure du peuplement, ecologie des populations de Chironomides. Thèse $3^{e}$ cycle, Université Aix-Marseille I. $196 \mathrm{p}$.

Origgi (L.), de Bernardi (R.) \& Giussani (G.). 1978.- Alcune osser vazioni sulla comparsa di Cyclops vicinus Ulja nin (Crastacea Copepoda) e sulla sua ecologia in alcuni laghi dell'Italia set tentrionale. Mem. Ist. Ital. Idrobiol 36:309 , 319

Patalas (X.). 1981 - Spatial structure of the crustacean plankto nic community in lake Wirnipeg. Canada. Verh. Intemat. Verein. Limnol. $21: 273 \cdot 279$.

Rigler (F-H.). 1973.- A dynamic view of the phosphorus cycle in lakes. In Environnemental Phosphorts Handbook. Ed. Griffith. Beeton, Spencer and Mitchel. J. Wiley and sons : 539 - 572.

Rylov (V.M.). 1963.- Freshwater Cyclopoida. Fauna of USSR, Vol 3, 3. Crustacea, $314 \mathrm{p}$.

Zurek (R.). 1980.- The effect of suspended materials on the zooplankton. Patural environments. Acta hydrobiol. 22 (4) : 449. 471. 